\title{
Una resignificación en los procesos de enseñanza del lenguaje y las matemáticas a partir de la implementación del Programa Todos a Aprender (PTA)
}

A new meaning in the teaching of language and mathematics from the implementation of the Program all Learn (PTA)

\section{Alex Mauricio Díaz Díaz \\ Universidad de Córdoba-Colombia alexmau995@gmail.com \\ Juan Gabriel Uribe \\ Universidad de Córdoba-Colombia jguribe@correo.unicordoba.edu.co}




\section{Resumen}

Este artículo presenta los resultados de un estudio de caso desarrollado con los docentes de básica primaria de la Institución Educativa "San José del Pantano" del Municipio de Puerto Escondido en el Departamento de Córdoba. Así mismo, se pretende demostrar cómo ha sido el impacto del Programa Todos a Aprender en los procesos de enseñanza del Lenguaje y las Matemáticas. La investigación se sustenta dentro del enfoque cualitativo, con un diseño metodológico de estudio de casos, focalizando en las categorías de comunidad de aprendizaje de docentes y conocimiento didáctico de los contenidos. Se desarrolló esta investigación en tres fases: conformación y consolidación de la comunidad de aprendizaje de docentes, seguimiento del aprendizaje y conformación de equipo de trabajo hacia la meta de la excelencia educativa. Se muestran los resultados obtenidos en términos de evaluación formativa del aprendizaje, acompañamiento en el aula y desarrollo profesional docente.

\section{Abstract}

This article aims to present the result from a case study applied in primary teachers at "San José del Pantano" School in Puerto Escondido - Córdoba. Which aims to assess the impact from "Todos a Aprender" Program in the processes of language and math teaching. This research has a qualitative approach with a case study design. Focusing in the categories of: Professionals Learning Communities, Content and Didactics Knowledge. For this investigation development it has been took into account three phases: Settle of a Professional learning community, Learning assessment and evaluation and team teaching for excellence and quality of education. Also, are shown the results gotten in the formative learning evaluation, formative assessment of learning support in the classroom and teacher professional development.

\section{Palabras Clave}

Lenguaje, Matemáticas, Comunidad de Aprendizaje, Evaluación Formativa, Seguimiento del Aprendizaje, Desarrollo Profesional Docente.

\section{Keywords}

Spanish, Math, Learning Community, Formative Learning Assessment, Teacher Professional Development. 


\section{Introducción}

El presente estudio se origina como una necesidad de reflexión sobre el impacto de la comunidad de aprendizaje de docentes en las prácticas de enseñanza del Lenguaje y las Matemáticas en básica primaria de la Institución Educativa "San José Del Pantano", a causa de los bajos resultados obtenidos por los estudiantes en las evaluaciones de pruebas nacionales externas como SABER - ICFES grados $3^{\circ}$ y $5^{\circ}$. Desde este objetivo de mejorar la instrucción y la práctica pedagógica, se pretende trabajar en las comunidades de aprendizaje los aspectos de conceptualización, retroalimentación y estrategias de evaluación en el desarrollo de las clases para garantizar el aprendizaje efectivo de los estudiantes. Todo este proceso educativo dará cuenta del mejoramiento de los resultados para las pruebas Saber $3^{\circ}$ y $5^{\circ}$ que se realizan en la Institución Educativa cada año. Lo cual se constituirá en uno de los factores para evaluar la calidad educativa que se está brindando en la institución.

Una de las metas de las políticas del gobierno actual es la de alcanzar para el año 2025 la calidad educativa, la cual puede ser concretada con prácticas de aula pertinentes, eficaces y significativas, ya sea en salones de clase o donde quiera que se desarrolle la acción pedagógica sistemática con el grupo de estudiantes. Nuestro sistema educativo estatal debe garantizar aprendizajes básicos, universalmente definidos como lo son las áreas de lenguaje y matemáticas las cuales son el sustento esencial para los demás aprendizajes dado que estás dos áreas se constituyen en los pilares de la educación básica primaria, secundaria, media y superior.

Luego entonces, las prácticas de aula se constituyen en una de las herramientas claves que contribuyen con la consecución del objetivo de la calidad educativa planteada por el estado. Una de las estrategias que mejoran las prácticas de aula y el proceso de enseñanza de los docentes es la implementación de comunidades de aprendizaje y de práctica. El proceso de formación docente debe, por consiguiente, dirigirse al colectivo de maestros y debe ser con formación situada, esto quiere decir, que debe desarrollarse en el contexto y en relación con la práctica de aula y sus problemáticas específicas. El aula forma parte de la estructura de la escuela como institución social y, por ende, no puede ser un espacio pedagógico aislado, debe ser un espacio abierto a la sociedad y a sus aportes. Por ello, el presente estudio se origina como reflexión acerca del impacto de la Comunidad de Aprendizaje ${ }^{1}$ en las prácticas de enseñanza del Lenguaje y Matemáticas de los docentes de primaria en la Institución Educativa "San José Del Pantano", a causa de los bajos resultados

\footnotetext{
${ }^{1}$ En lo sucesivo del texto, se utilizará la abreviatura CDA para referirse a las Comunidades de Aprendizaje.
} 
obtenidos por los estudiantes en las evaluaciones de pruebas nacionales externas como SABER - ICFES grados $3^{\circ}$ y $5^{\circ}$ en las áreas mencionadas.

Las comunidades de aprendizaje son grupos que trabajan en torno de un propósito común; en este caso mejorar el aprendizaje, el rendimiento académico de los estudiantes y la instrucción del docente (Nunan, 2003). Una CDA es autodidacta y crece en conocimiento y capacidad continuamente, para tener mejores herramientas que se puedan utilizar en su plan de acción. Puede estar conformada por los docentes de cualquier nivel de educación; primaria, básica y media de las Instituciones Educativas del sector oficial o privado y Urbano o Rural. Una CDA promueve la equidad y pertinencia en los procesos educativos con participación de la comunidad en general. La CDA fomenta la contextualización de la educación a las realidades socioculturales de cada comunidad. De aquí que se utilicen, principalmente, como técnicas de recolección de información y de trabajo, las encuestas, las entrevistas, los cuestionarios y la observación directa.

El desarrollo de este estudio permitiría además, a propósito de las etapas de formación y evaluación de la Comunidad De Aprendizaje, actualizar a los docentes en las nuevas estrategias pedagógicas y en el uso de los materiales educativos y herramientas didácticas para el mejoramiento del desarrollo de sus clases, lo cual conlleva también a mejorar el bajo desempeño de los estudiantes en sus niveles de aprendizaje de las áreas de Lenguaje y Matemáticas,, ya que los estudiantes y docentes no están haciendo un uso pertinente del material didáctico recibido. La etapa de evaluación se definirá como la valoración del trabajo desarrollado por toda la CDA en el establecimiento educativo y su impacto en: estudiantes, docentes y padres de familia de la comunidad estudiantil. Estableciendo, si se pudo alcanzar o no con la transformación de los procesos de enseñanza - aprendizaje y el mejoramiento de la calidad educativa de los estudiantes en las áreas de Lenguaje y Matemáticas en los diferentes niveles de la educación básica primaria. Las anteriores consideraciones permiten la formulación de la siguiente pregunta problémica:

¿Cuál es el impacto del Programa Todos a Aprender en los procesos de enseñanza del Lenguaje y las Matemáticas en la Institución Educativa "San José del Pantano"?

\section{Antecedentes}

Las Comunidades de Aprendizaje de Docentes se constituyen en uno de los logros fundamentales derivados de las experiencias internacionales de los mejores sistemas educativos de países como Finlandia, Singapur y Canadá. Así, en el ámbito internacional se encuentran las investigaciones realizadas en el ámbito americano. Slavit, investigador norteamericano de la Universidad de Delaware, ha trabajado temáticas relacionadas con la formación docente, la instrucción y la enseñanza colaborativa, mostrando entre los resultados 
obtenidos, el impacto en la transformación de las prácticas educativas y en la calidad del aprendizaje de los estudiantes mediadas por la metodología de estudio de clases $^{2}$ (MEC). En el año 2008, llevó a cabo el proyecto “Professional Learning Communities" (PLCs) para mejorar la enseñanza de las matemáticas en los niveles de básica primaria y secundaria a través de la vivencia de Comunidades de aprendizaje. Así, estableció criterios y parámetros para el desarrollo significativo de estas formas de organización.

En el mismo contexto norteamericano, se encuentra también el Dr. David Nunan, docente de la Universidad de Anaheim en California, quien ha investigado sobre la enseñanza colaborativa y las comunidades de aprendizaje de docentes, en un proyecto reseñado como "Estudio de caso sobre el modelo educativo Japonés" (1993), desde el British Council, Cambridge Center y Ministerio de educación nacional Japonés. En este estudio se investigó acerca de la implementación de comunidades de aprendizaje de docentes y la Metodología del Estudio de clases cómo ejes fundamentales para alcanzar procesos de calidad educativa a través de: 1. Desarrollo de procesos de investigación educativa cualitativa para la implementación de estrategias metodológicas pertinentes y significativas de acuerdo con el contexto y las necesidades educativas especiales de los estudiantes. 2. Equipos de estudio de clases (MEC) donde se observa, evalúa y retroalimenta el proceso de enseñanza de cada uno de los docentes participantes en la comunidad de aprendizaje, y 3. Construcción conjunta, encaminada a mejorar las prácticas pedagógicas a través de una visión colectiva y colaborativa de los procesos que se están desarrollando actualmente en los establecimientos educativos (Nunan, 1993).

Las conclusiones que se abordan del proceso investigativo de Nunan (1993) están relacionadas con: 1. Enfocarse en objetivos comunes, más que en personalidades individuales. 2. Reconocer mutuamente las contribuciones y aciertos en el logro de los objetivos. 3. Preparar un tiempo y lugar específico para planear y preparar todo para las clases. 4. Manejar las reuniones de planeación con tiempos y objetivos claros para evitar perder el tiempo o quedarse en discusiones innecesarias. Además, las actividades colaborativas permiten ampliar el rango de interacción y trabajo en el aula y posibilitan acercarse a un problema o una situación haciendo conexiones cognitivas o sociales de manera diversa y diferenciada lo que enriquece los procesos cognitivos, afectivos, comunicativos, y por supuesto la interacción social (Nunan, 2003).

Dentro del marco español, se resalta la investigación liderada por Valls Carol (1999) denominada "Comunidades de Aprendizaje: Una Práctica educativa de Aprendizaje dialógico para la sociedad de la información", estudio que se orientó hacia el análisis del proceso necesario para la conformación, consolidación y proyección de una CDA de docentes a partir de las fases de sensibilización, toma de decisiones, sueño, selección de prioridades,

\footnotetext{
${ }^{2}$ En lo sucesivo del texto, se utilizará la abreviatura MEC.
} 
planificación, investigación, formación y evaluación. Además, resalta la importancia del contexto para la atención de las necesidades educativas de los estudiantes y el mejoramiento de la calidad de la enseñanza de los docentes.

En el ámbito Latinoamericano se encuentra la "Estrategia LEM De Chile"; Experiencia Educativa de Campaña De Lectura, Escritura y Matemática, liderada por el Ministerio de educación de ese país desde el año 2002, donde se tiene como objetivo general el aprendizaje colaborativo y la reflexión de la práctica educativa; el mejoramiento de los aprendizajes de los estudiantes en las áreas de Lenguaje y Matemática, desde preescolar hasta la Educación Básica (9 años de edad), por medio de la cualificación de las competencias y conocimientos didácticos de los docentes las cuales fueron escogidas dada su relevancia en la comprensión de las otras áreas del saber y las habilidades que implica su aprendizaje para analizar, razonar y participar en los diferentes dinámicas de la sociedad (Vezub, 2009). Esta política educativa pretende mejorar el rendimiento académico de los estudiantes, así como los índices de deserción y repitencia, fundamentada en tres aspectos claves: la elaboración de unidades didácticas de lenguaje y matemática con estrategias pedagógicas diseñadas por expertos, el desarrollo profesional de los docentes; y el acompañamiento del trabajo didáctico en las aulas de clase.

Dentro de la estrategia LEM liderada por el ministerio De Educación Chileno se han alcanzado los siguientes resultados según (Vezub, 2009): “Formación de los profesores consultores, talleres de estudio institucionales en torno a la discusión y análisis de la unidad didáctica diseñada por especialistas, talleres inter-institucionales que reúnen a tres escuelas de la zona, Trabajo "in situ" entre el profesor del aula y el Profesor consultor (PC), talleres de retroalimentación o devolución al finalizar el desarrollo de cada unidad didáctica".

En nuestro país, la implementación de la metodología del estudio de clases como propuesta de trabajo en CDA se ha desarrollado en diferentes regiones del país tales como la Región Andina y del Caribe, entre otras. Cuyo objetivo o propósito principal era la implementación de experiencias educativas de docentes trabajando en equipos de estudio para mejorar la enseñanza de las matemáticas en los niveles de la educación básica primaria a partir de la conformación de una CDA en cada Institución Educativa focalizada. Mediante, la Metodología del Estudio de clases donde se realizaron ejercicios de: Planeación, ejecución, observación y evaluación de las clases en equipos de estudios (CDA). De esta forma, los resultados obtenidos en esta experiencia educativa fueron: la ayuda de los demás compañeros a mejorar la instrucción. En la experiencia "Maestro invítame a tu Clase" de manera sencilla y tranquila se observó y aprendió del otro. También, se trabajaron las clases demostrativas, donde "un buen grupo de profesores ayuda a un compañero a preparar con anticipación un tema determinado, para luego trabajarlo con los estudiantes y allí evaluar y mejorar cada día" (Antioquia: 2007). 
Las investigaciones y experiencias reseñadas en este apartado demuestran la preocupación y el interés de todos los actores del sistema educativo. Gobiernos y Establecimientos Educativos, entre otros, por lograr el mejoramiento de la instrucción, de la enseñanza y del aprendizaje de competencias necesarias para el mejoramiento de la calidad educativa a nivel regional, nacional e internacional desde la educación inicial en las áreas de lenguaje y matemáticas. Por lo tanto, los anteriores estudios permiten concluir que las investigaciones encontradas están orientadas hacia el aprendizaje cooperativo, colaborativo, desarrollo profesional situado y pedagógico de la siguiente manera:

- Desarrollo de procesos participativos que desembocan en propuestas practicas mediante métodos cualitativos donde la participación de la comunidad de aprendizaje permite el desarrollo de procesos académicos y culturales como base para incidir también, en la transformación de la calidad educativa donde se ejecutan estos tipos de sistemas educativos.

- Este tipo de programas educativos en su gran mayoría buscan mejorar la práctica educativa y el desarrollo de habilidades y competencias en los estudiantes a través del trabajo en equipo de los docentes y de la aplicación de la metodología del estudio de las clases en las áreas fundamentales del saber.

- Existe una preocupación generalizada por el mejoramiento de la instrucción y de la práctica educativa dentro del aula de clases donde se desarrollen estrategias y metodologías acordes con el ritmo de aprendizaje de los estudiantes basados en las competencias y el potenciamiento de habilidades y destrezas en las áreas del Lenguaje y las Matemáticas.

- En términos metodológicos la mayoría de investigaciones son cualitativas de carácter mixto, que proponen la enseñanza mediante el aprendizaje colaborativo y cooperativo entre pares y comunidades de aprendizaje que planean, desarrollan, observan y evalúan las clases entre todos.

Con la metodología del "Estudio de Clases" implementadas en el país, la formación profesional docente y la atención de la educación básica primaria se dio inicio a la implementación del Programa Todos a Aprender en el país focalizando aspectos: de pedagogía donde se privilegia la interacción comunicativa que se establece entre el docente y los estudiantes en contextos específicos. De gestión desde un enfoque sistémico que facilita las relaciones entre los diferentes agentes y niveles, al tiempo que identifica y optimiza los recursos nacionales, regionales, institucionales y del entorno para imprimirle efectividad, sostenibilidad y eficacia a la estrategia pedagógica del PTA ${ }^{3}$ (MEN: 2012). Por último, de formación situada sustentada en el acompañamiento en el

\footnotetext{
${ }^{3}$ En lo sucesivo del texto se utilizara PTA para hacer referencia al Programa Todos a Aprender.
} 
aula a los docentes y en las problemáticas específicas del aula de clase en torno a los procesos de aprendizaje de los estudiantes, enfocada en la estructuración de oportunidades para que el colectivo de maestros afine sus prácticas de aula; todo esto, con el objetivo de mejorar los niveles de desempeño de los estudiantes de las instituciones educativas del sector oficial ubicadas en zonas urbanas y rurales en las áreas de Lenguaje y Matemáticas en el nivel de la educación básica primaria.

Shulman establece qué: "el conocimiento didáctico del contenido 4 incluye las conexiones entre los conocimientos de la materia y didácticos del profesor" (2005). Donde, la importancia de poseer el dominio didáctico y curricular de la asignatura que se imparte, la facultad de presentar diversas alternativas a la hora de realizar una clase y desarrollar el proceso de enseñanza- aprendizaje, permite que este sea más significativo para todas los participantes del entorno educativo es decir; estudiantes, docentes, y padres de familia. Por lo tanto, es necesario tener presente a todos los actores involucrados y los diferentes escenarios que conforman la dinámica educativa. Donde, "se pueda evidenciar lo significativo de poseer un dominio de lo que se propone enseñar, pero también la didáctica para enseñarlo de la forma más efectiva posible" (Shulman, 2005).

En lo que respecta al conocimiento de la didáctica general, esta repercute en el análisis efectivo de la práctica permitiendo al docente conocer la evolución que ha tenido la educación y sus diferentes concepciones en cada época, hasta nuestros días. Con este análisis el docente puede entender los aciertos y desaciertos de la práctica pedagógica y retomar lo que tiene valides para él, teniendo en cuenta las características de sus estudiante y su contexto social y cultural.

El manejo del CDC permite al docente establecer interacción entre la materia y la didáctica, dando lugar a la transposición, "que es transformar el conocimiento de la materia en formas que sean didácticamente impactantes y se adapten a la variedad que presentan los estudiantes en cuanto a ritmos de aprendizaje, habilidades y bagaje" (Chevallierd, 1985). De allí la importancia de conocer la didáctica especifica de un contenido, con el fin de buscar el camino adecuado desde la comprensión de la materia por parte del profesor hasta llegar a la motivación y percepción de los alumnos, ya sea empleando analogías, ilustraciones, explicaciones, ejemplos o demostraciones. En lo concerniente al conocimiento de los alumnos y sus características, esta categoría permite al docente implementar estrategias de adaptación; adecuando el material a trabajar a las características del alumno, bien sea teniendo en cuenta sus capacidades, cultura, conocimientos previos, ritmos de aprendizaje y motivaciones, entre otros. Resaltando la importancia, que para el docente tiene; conocer los procesos sicológicos del aprendizaje de los alumnos de acuerdo a su

\footnotetext{
${ }^{4}$ En lo sucesivo del texto se utilizará CDC, para hacer referencia al Conocimiento Didáctico Del Contenido.
} 
edad y sus ritmos y estilos de aprendizaje además de las preferencias para un determinado tema, para de este modo poder ser efectivos al momento de enseñar. Es importante, realizar este razonamiento para la acción pedagógica, puesto que se tienen en cuentan las verdaderas necesidades e intereses del educando. Un buen conocimiento del alumnado permite interpretar mejor sus ideas y organizar la enseñanza con mayor eficacia.

\section{Metodología}

Este proyecto investigativo tiene un enfoque cualitativo y su propósito es, conceptualizar sobre la realidad con base en el comportamiento, los conocimientos, las actitudes y los valores que guían el comportamiento de las personas estudiadas. El proceso de investigación cualitativa explora de manera sistemática los conocimientos y valores que comparten los individuos en un determinado contexto espacial y temporal" (Bonilla, 2005). En este orden de ideas, la implementación de este tipo de enfoque cualitativo en nuestro proyecto investigativo ha permitido conocer directamente la realidad social de cada uno de los actores del contexto educativo. Así mismo, una mayor apreciación frente la comunidad educativa de docentes, estudiantes y padres de familia con el objetivo de brindar soluciones a las necesidades de cada uno de estos actores en el proceso educativo.

Se configura un estudio de casos con un abordaje crítico social, para posibilitar la intervención aspirando transformar los procesos de enseñanza, pasando de la clase magistral a la Metodología del Estudio de Clases (MEC), con equipos de estudio de docentes para el aprendizaje colaborativo y modelos educativos centrados en el estudiante en el contexto de la Institución Educativa San José Del Pantano. De esta forma, se aproxima a la particularidad, singularidad y heterogeneidad de la práctica de los docentes desde tres momentos: antes de que suceda (planeación de la clase), durante su desarrollo (saber práctico de los docentes) y después que suceda (reflexión y revisión de la práctica) (Litwin, 2008).

El estudio de casos se centra en la conformación de un equipo de docentes, quienes se han planteado un plan de trabajo focalizado sobre un problema concreto de aula como comprensión de contenidos, uso del material didáctico y las interacciones maestro - estudiantes en la clase, entre otros. Con el fin de asegurar la pertinencia del proceso de enseñanza y la efectividad en la práctica, de cara al cumplimiento de los objetivos propuestos por el Ministerio de Educación Nacional y el Programa Todos a Aprender. De esta forma, se lleva a cabo un estudio de la particularidad, singularidad y heterogeneidad de la práctica de los docentes desde tres fases: proyección de las comunidades de aprendizaje, ejecución del desarrollo profesional situado y consolidación de la 
comunidad de aprendizaje de docentes donde se aplica la reflexión y revisión de la práctica pedagógica constantemente (Valls, 2005).

En el desarrollo de este estudio, se asume una perspectiva metodológica de tipo evaluativo, lo cual supone un "proceso de recogida de información orientado a la emisión de juicios de mérito o de valor respecto de algún sujeto, objeto o intervención" (Casarrubios et al., 2009, p. 2). Como marco orientador del estudio, la investigación permite un análisis del impacto del programa Todos a Aprender, en especial, de la comunidad de aprendizaje en la práctica de enseñanza del lenguaje y las matemáticas de los docentes de la Institución Educativa San José del Pantano.

La población de esta investigación está compuesta por los dieciséis profesores de básica primaria de la Institución Educativa San José Del Pantano. La muestra está compuesta por todos estos docentes de básica primaria del establecimiento educativo. A causa del número reducido de maestros de la Institución Educativa en el nivel de básica primaria, se ha optado por elegir como muestra la totalidad de docentes de este nivel educativo, que constituyen la comunidad de aprendizaje con la cual se está trabajando.

Las técnicas de investigación utilizadas en el presente estudio son el cuestionario, la observación participante y la entrevista a profundidad. La observación participante es un elemento fundamental en los estudios de casos, es una técnica implícita en todas las técnicas utilizadas. La observación permite identificar el pensamiento del docente a través de la admiración de las acciones, por lo que se puede comprender mirando desde afuera lo que desde nuestro punto de vista parecía visible. Con la observación, se puede describir el quehacer docente en los procesos de enseñanza del lenguaje y las matemáticas, así como reconocer su realidad académica. Con este recurso metodológico, se recolecta información sobre las acciones que realizan los docentes cotidianamente, dentro o fuera de un aula de clases.

"La operación del mirar implica otra, la de ad-mirar. Admiramos y, al adentrarnos en el admirado, lo miramos de dentro y desde adentro, lo que nos hace ver". Castellanos (2012).

Desde allí se parte hacia un proceso de comprensión y posterior resignificación del actuar en el proceso de enseñanza. Con esta técnica, se observan los hechos en forma no estructurada, se recogen impresiones generales de los sucesos, procesos, acontecimientos o situaciones naturales producidos espontáneamente en el entorno.

La entrevista a profundidad es una herramienta clave para conversar individualmente con los docentes acerca de criterios y valoraciones del accionar. Para conocer los pensamientos y acciones de una persona, hay que hablar sobre la vida y la carrera. Lo anterior es una base para investigar sobre la historia de vida del docente, cómo empezó enseñando, su evolución y cómo enseña actualmente. Las experiencias educativas positivas y los intereses principales de 
los docentes (Woods, 1998). Por su parte, los instrumentos de investigación usados son el diario de campo, la grabadora y la cámara de video.

\section{Resultados}

En el marco de esta investigación se realizó un análisis de tres aspectos que se focalizan dentro del Programa Todos a Aprender, aplicado en la Institución Educativa San José Del Pantano desde el segundo semestre del año 2013. Los cuales son: evaluación formativa del aprendizaje, acompañamiento en el aula y desarrollo profesional docente. Desde el enfoque de pertinencia, confiabilidad y transparencia para la obtención de la información desde los diferentes actores como docentes, directivos docentes y evaluadores externos. Además, una compilación de información, donde la intención es evaluar el impacto del programa en los aspectos de mejoramiento de la calidad educativa y la instrucción docente en la institución educativa focalizada.

\section{Evaluación formativa del Aprendizaje.}

Los docentes de Educación básica Primaria de la Institución Educativa constantemente desarrollar actividades de seguimiento y de evaluación formativa del aprendizaje de los estudiantes en las áreas de Lenguaje y Matemáticas, a través de evaluaciones diagnosticas formativas, instrumentos de evaluación formativa tales como rubricas, listas de cotejo y guías de aprendizaje, entre otros. Lo cual, ha evidenciado un comportamiento ideal para las aspiraciones institucionales de la Institución Educativa, donde se puede decir que, en primer lugar, se cumplieron las metas planteadas por el programa Todos a Aprender de mejorar las condiciones de aprendizaje para los estudiantes matriculados entre transición y quinto grado de básica primaria. En segundo lugar, 25 \% de los estudiantes de la Institución Educativa San José del Pantano debían ascender del nivel insuficiente en los resultados de las pruebas Saber 2014, lo cual se alcanzó satisfactoriamente en los resultados de las evaluaciones externas - Saber 2014. Tal como se muestra en el siguiente gráfico: 
Gráfico 1. Resultados Pruebas Saber 2014

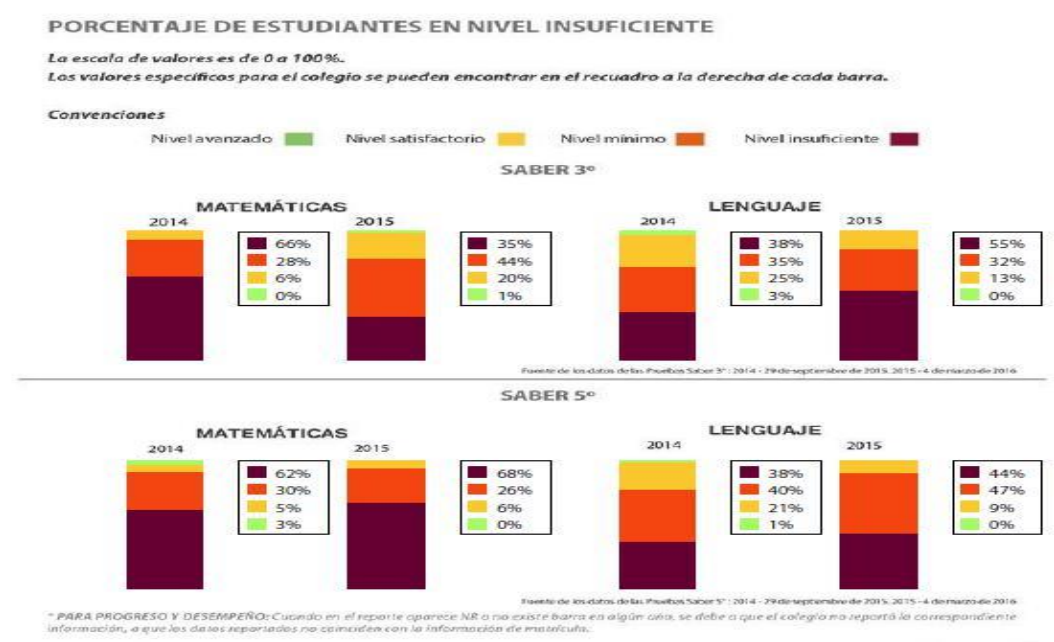

Fuente: http://diae.mineducacion.gov.co/dia_e/documentos/2016/223574001314.pdf

\section{Acompañamiento en el aula.}

De acuerdo con los lineamientos del Ministerio de Educación Nacional y las orientaciones pedagógicas del Programa Todos a Aprender: “El acompañamiento en el aula (Véase Gráfico 2), es una estrategia privilegiada que propende por la interacción entre pares, donde el tutor, como par y acompañante externo de la práctica, puede ayudarle al docente a identificar oportunidades de mejoramiento hacia la transformación de las prácticas de aula. Esta interacción permite generar procesos de evaluación formativa, a través del reconocimiento de las estrategias, acciones y actividades que garanticen el mejoramiento de los aprendizajes en Lenguaje y Matemáticas de los estudiantes que forman parte de establecimientos educativos focalizados por el Programa Todos a Aprender" (MEN - PTA: 2016).

Gráfico 2. Modelo de Acompañamiento en el aula.

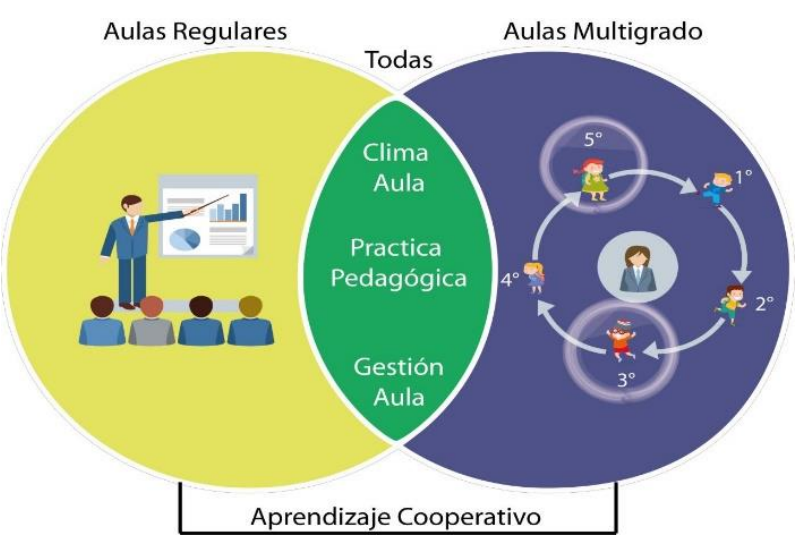

Fuente: Ministerio de Educación Nacional de Colombia, Programa Todos a Aprender 2.0, Lineamientos acompañamiento en aula 2016. 
Así mismo, se ha encontrado que el principal objetivo del acompañamiento en aula al docente es el resultado significativo que se obtiene en el mejoramiento de los aprendizajes de los estudiantes en las áreas de Lenguaje y Matemáticas a través de la transformación de las prácticas de aula implementadas por el docente atendiendo los criterios de; acciones didácticas de contenido de la clase, gestión de aula, evaluación formativa y uso de material educativo entregado por el Programa todos a aprender.

\section{Desarrollo Profesional Docente.}

La formación pedagógica y actualización docente en el programa Todos a Aprender se centra en dos estrategias didácticas del Aprendizaje: aprendizaje colaborativo y aprendizaje cooperativo. El aprendizaje colaborativo desarrolla la participación, es decir, implica la conformación de pequeñas comunidades de aprendizaje. En este proyecto investigativo, se encontró que los docentes del área de humanidades fortalecieron sus debilidades en la didáctica de las matemáticas con el apoyo y sustento de los dos docentes licenciados en matemáticas, quienes durante las sesiones de trabajo situado en comunidades de aprendizaje retroalimentaron el proceso metodológico de las enseñanzas de las matemáticas basados en los procesos y pensamientos matemáticos, así como los docentes en humanidades fortalecieron el quehacer pedagógico en las prácticas educativas de lenguaje.

En las prácticas colaborativas, los docentes se benefician, porque disminuyen su aislamiento $\mathrm{e}$ incertidumbre y conforman verdaderas comunidades de apoyo, más productivas y críticas sobre las prácticas, hacia una verdadera gestión del cambio, con la actualización pedagógica que se trabaja en los equipos de estudio y las sesiones de trabajo situado, donde se observan las clases y se debate en torno a una problemática previamente establecida. Es decir, gestión del tiempo del aula, ambiente escolar, conocimiento didáctico de los contenidos y estrategias pedagógicas eficaces y efectivas para la instrucción docente, que demuestran un proceso significativo de la instrucción y del quehacer docente en nuestras aulas de clases de la Institución Educativa San José del Pantano.

En segundo lugar, se pueden rescatar como resultados de la formación docente el liderazgo, el sentido de responsabilidad que se despierta por el aprendizaje propio y el de los demás, la tarea como producto de una acción conjunta del grupo, el análisis e identificación de las formas como se aprende y aprenden los demás y la compañía del docente como observador de las dinámicas, mediador en el aprendizaje y orientador de las acciones promovidas para la enseñanza a partir de las experiencias adquiridas por el papel docente o la formación in situ que se trabaja durante las reuniones semanales dentro de las orientaciones del programa Todos a Aprender, donde se desarrollan aspectos de pedagógica, didáctica y evaluación mediante simulaciones de clases con juegos de roles de los docentes participantes. 
Por último, estas prácticas promueven el desarrollo profesional y la afirmación de la identidad del maestro y su lugar como miembro valioso de la comunidad educativa. El trabajo colaborativo y cooperativo de los profesores en los procesos de planeación, enseñanza y evaluación promueven mejores prácticas pedagógicas, mejoran el clima institucional y promueven la creatividad y el desarrollo profesional docente. Además, el poder de la observación como acompañamiento a clase para explorar el estudio de clase y la práctica y sus protagonistas como objeto de reflexión e investigación.

\title{
Discusión
}

En la ejecución del PTA merecen especial atención algunos aspectos los cuales deben ser atendidos oportunamente. Tales como, el fortalecimiento de las relaciones entre docentes, alumnos y padres, ya sea a través de la implementación de escuela de padres o de Comunidades de Aprendizaje de Padres de Familia donde se orienten proyectos acerca del acompañamiento al proceso de aprendizaje de sus hijos o educando y de seguimiento del aprendizaje de los estudiantes mediante la triangulación de las relaciones interpersonales de Docentes, Estudiantes y Padres de Familia. De acuerdo con Díaz:

\begin{abstract}
El papel que debe darse a la formación ciudadana y al uso de las nuevas tecnologías es fundamental en la ejecución del Programa Todos a Aprender, dado que estos factores no tienen visibilidad en el programa, ya que se han priorizado otros elementos, cuyo tratamiento parece ser más apremiante en aras de garantizar la base sobre la cual empezar a trabajar otros aprendizajes. Además, el Ministerio cuenta con otros programas que apuntan a la calidad no solo en función de lo cognitivo sino también de lo actitudinal. (p.10).
\end{abstract}

En consonancia con lo anterior, se puede decir que el Ministerio de Educación Nacional y las orientaciones pedagógicas del equipo de formadores y docentes tutores del PTA atiende algunas características básicas como el reconocimiento del contexto social específico en el que se acompaña a los docentes y a los Establecimientos Educativos focalizados, comprende dimensiones clave de la calidad como la equidad, relevancia y pertinencia de los procesos de enseñanza por parte de los docentes atendiendo acciones didácticas y orientaciones pedagógicas centradas en el aprendizaje y los estudiantes; y finalmente, da cuenta de aspectos generalmente ligados a la calidad, como lo es el uso de materiales educativos pertinentes y significativos para el aprendizaje del Lenguaje y Matemáticas; por lo que se puede decir que el PTA tiene gran relevancia en el país y en el sector educativo oficial, lo cual es evidente en el contexto en que emerge en este caso en una zona rural dispersa con condiciones sociales difíciles y de poco acompañamiento comprometido de 
los entes territoriales certificados y de las secretarias de educación para contribuir con el logro de la calidad educativa ya que se constituyen en nuestros orientadores del proceso educativo y coordinadores pedagógicos de los lineamientos pedagógicos para cada institución.

\section{Conclusiones}

De este proyecto investigativo, se derivaron algunas conclusiones entre las cuales se tiene; las Comunidades de Aprendizaje se han convertido en una herramienta de trabajo en equipo para la enseñanza colaborativa y la cualificación docente permitiendo el mejoramiento continuo, ya que los docentes se constituyen en un par académico que evalúa los proceso de enseñanza de los docentes a través de la ejecución de la MEC. La participación activa de docentes, directivos docentes, estudiantes y padres de familia fortalecen el proceso de enseñanza - aprendizaje puesto que se crea un espacio de comunicación permanente para el mejoramiento del rendimiento académico del estudiante, del acompañamiento del padre de familia y del proceso educativo del docente, todo esto bajo la mirada reflexiva del directivo docente quien funciona como orientador de los procesos académicos, curriculares y administrativos. Atendiendo las características básicas del contexto social específico en el que se encuentran las instituciones educativas del municipio de Puerto Escondido - Córdoba. Destacando aspectos claves de la calidad como la equidad, relevancia y pertinencia.

En el uso de materiales educativos para el desarrollo del área de Lenguaje y Matemáticas, se destaca la motivación constante por parte del estudiante para estar en las sesiones de trabajo de estas dos áreas lo que es justificado por los docentes como resultado de las actividades de formación situada y la labor de las Sesiones de Trabajo Situado, y el acompañamiento en el aula por parte de la CDA y de los procesos de planeación con los docentes integrantes del equipo MEC. Además, el mejoramiento de la instrucción del docente en estas áreas ya que se utilizan estrategias pedagógicas centradas en el estudiante y bajo las orientaciones pedagógicas de los estándares básicos de competencias, los derechos básicos de aprendizaje y los lineamientos curriculares para la enseñanza de estas dos áreas fundamentales del proceso educativo. 


\section{Referencias}

\section{Bibliográficas}

Agudelo, J.D. (2007). Estudio de clase: Estudio de áreas y perímetro dentro de una cuadricula haciendo uso de la tecnología. Estudio de Clase no publicado Escuela Normal Superior María Auxiliadora, Granada - Meta, Colombia.

Castellanos C. (2012). Los pensamientos de los profesores universitarios. Las concepciones pedagógicas sobre la enseñanza y el aprendizaje en la facultad de ciencias de la salud. Maestría en Educación SUE CARIBE. Universidad de Córdoba. Montería. Colombia.

Chile, M. d. (2001). Estándares de Desempeño para la Formación Inicial de Docentes. Santiago De Chile: División de Educación Superior.

Freire, P. (1993). Pedagogica de la Esperanza. Mexico: Editorial Siglo XXI. (Trabajo Orignial publicado en 1992).

Furman, M. 2012. Orientaciones técnicas para la producción de secuencias didácticas para un desarrollo profesional situado en las áreas de matemáticas y ciencias. Ministerio de Educación Nacional.

Gana A., C., Lee O., M., Galdames F., V., \& Concha Carreño, L. (2006). Asesoría a la Escuela para la. Ministerio de Educación, República de: División de Educación General.

Habermas, J. (1993). Teoria de la Acción Comunicativa: Complementos y Estudios

Previos. Madrid: Cátedra. (Trabajo Orignial publicado en 1984)

Instituto para la Cooperación Internacional. Agencia de Cooperación Internacional del Japón (JICA) (2006) Maestros aprendiendo juntos. [Material audiovisual].

Marco para la Buena Enseñanza (2003). Centro de Perfeccionamiento, Experimentación e Investigaciones Pedagógicas (CPEIP) Ministerio de Educación. República de Chile.

McKinsey. (2007). How the world's best-performing school systems come out on top.

MEN. (2011). Ruta metodológica para el mapeo, documentación y análisis de buenas Prácticas en la promoción de los derechos sexuales y reproductivos. Bogotá: UNFPA-MEN.

Metodología Estudio De Clase [diagrama No1]. (2009) Recuperado de Curso b-learning en Metodología Estudio de Clase.

Ministerio De Educación Nacional. (2006). Estándares básicos de competencias en lenguaje, matemáticas, ciencias y ciudadanas. Colombia: Bogotá.

Ministerio de Educación Nacional (2012). Propuesta para la consolidación de los módulos en didáctica del Lenguaje y las Matemáticas en el marco del Componente de formación situada.

Ministerio de Educación Nacional (2009). Estudio de clase: una experiencia en Colombia para el mejoramiento en las prácticas educativas.

Nunan, D. (2003). Collaborative Language Learning and Teaching. Cambridge: Cambridge University Press.

Peter Woods, (1998). El arte de enseñar. Investigar el arte de la enseñanza, el uso de la etnografía en la educación. $1^{\mathrm{a}}$ edición. Barcelona: Paidos Ibérica S.A. 
Una resignificación en los procesos de enseñanza del lenguaje y las matemáticas a partir de la implementación del Programa todos a Aprender (PTA)

\section{Assensus}

Revista de Investigación educativa y pedagógica

\section{Electrónicas}

Diaz, Barreira \& Pinheiro (2015) Evaluación del programa Todos a Aprender: Resultados de la evaluación de contexto. Revista de estudios e investigación en psicología y educación ISSN:
2386-7418,
2015 ,
vol.
Extr.,
no.
10. Recuperado
de:

http://revistas.udc.es/index.php/reipe/article/view/360/pdf_265

Elizarrás, G. (2010). Las comunidades de aprendizaje: ¿Una alternativa para la educación actual? Disponible en http://www.cchep.edu.mx/docspdf/cc/109.pdf.

Slavit, D. (2008). http://www.teqjournal.org/. Recuperado el 31 de Marzo de 2014,http://www.teqjournal.org/Back\%20lssues/Volume\%2035/VOL35\%20PDFS/35_1/14 nelson\&slavit-35_1.pdf

Shulman, L. S. (1987). Knowledge and teaching: foundations of the new reform.

Harvard Educational Review, 57(1), 1-22. Traducción castellana (2005): Conocimiento y enseñanza: fundamento de la nueva reforma. Profesorado. Revista de Currículum y Formación de Profesorado, 9(2), http://www.ugr.es/ recfpro/rev92ART1.pdf.

Valls, R. (06 de Septiembre de 2005). Revista de educación social. Recuperado el 31 de marzo de 2014, de Revista de educación social: http://www.eduso.net/res/?b=7\&c=53\&n=145

Vezub, L. F. (11 de Noviembre de 2009). Organización de las Naciones Unidas para la Educación, la Ciencia y la Cultura (Unesco). Obtenido de Instituto Internacional de Planeamiento de la Educación (IIPE): http://www.iipe-buenosaires.org.ar 